Proceedings of the 2009 Winter Simulation Conference

M. D. Rossetti, R. R. Hill, B. Johansson, A. Dunkin, and R. G. Ingalls, eds.

\title{
IMPACTS OF RADIO-IDENTIFICATION ON CRYO-CONSERVATION CENTERS THROUGH SIMULATION
}

\author{
Sylvain Housseman \\ Nabil Absi \\ Dominique Feillet \\ Stéphane Dauzère-Pérès \\ Ecole des Mines de Saint-Etienne \\ Centre Microelectronique de Provence \\ 13120, Gardanne, FRANCE.
}

\begin{abstract}
This paper deals with using simulation as a decision support tool for estimating the impact of RFID technologies within biological sample storage areas (called biobanks). Several indicators, including inventory reliability or human resource utilization, are compared and discussed for different scenarios of use of the technologies. A special emphasis is put on the so-called re-warehousing activity that RFID makes possible, and which consists in reassigning tubes to empty places when box are emptied. Optimization algorithms are developed and embedded in the simulator. Results demonstrate the potential interest of RFID in biobanks and the value of simulation for estimating and optimizing such complex socio-technical systems.
\end{abstract}

\section{INTRODUCTION}

Trying to estimate the impact of introducing auto-IDentification technologies in a logistic process is a complex topic. The possibilities offered by these technologies may indeed affect the process in many different ways, at several decision levels, sometimes even enabling drastic changes. Simulation offers a practical tool for addressing these questions without referring to costly and very focused pilot studies. This paper proposes to use simulation for estimating the impact of introducing RFID technologies within biobanks. This study is a part of the so-called MISTRALS project, involving a couple of industrial companies and two French health structures developing a biobank activity: the Institute Paoli-Calmettes (IPC) in Marseilles, and the $\mathrm{CHU}$ of Nice (CHU-N).

Biobanks, or more generally Biological Resource Centers receive, transform, store and make available biological (human) samples and their derivatives. The biobanks considered in this study stock samples at very low temperatures $\left(77^{\circ} \mathrm{Kelvin}\right)$, in Nitrogen tanks. Samples are intended to be used within research programs and for diagnosing or evaluating the risk to develop cancer. Biobank activity can be regarded as intermediate between stock management and health delivery. Like most of the health production systems, many human manipulations are required. Traceability is undoubtfully of essential importance for biobanks and Smart objects are natural candidates for contributing to improvements in this direction.

Smart objects are objects that have been tagged with an RFID transponder (or tag), and thus can transfer and record information without any human action. RFID is the generic acronym for Radio Frequency IDentification (or Radio Frequency Identification Devices). It basically works as follows: an RFID base-station antenna sends electromagnetic waves that are captured by the RFID tag's antenna. These waves allow to empower the tag's chip, which modifies the signal. The reader's antenna decrypts the modifications and thus deduce the information embedded in the tag (Hedgepeth 2007). Depending on the technology, different frequency bands, ranging from Low Frequency (125 kHz) to Super High Frequencies $(5.8 \mathrm{GHz})$, can be used with different data transfer rates and reading capabilities. New generations of RFID tags, readers and protocols allow a large amount of data (a few kilobytes) to be embedded, multiple reading and writing, on-chip sensors (Miles, Sarma, and Williams 2008) and objects to communicate with each other. The increasing utilization of RFID and the appearance of standards and protocols allow producers to lower tag prices and users to label items at a lower additional cost.

A prominent domain of application for RFID technologies is supply chain management (Saraç, Absi, and Dauzère-Pérès 2008a). Applications are numerous including simple display of information, recording of statistics for planning and scheduling, traceability, inventory management, order verification, quality control. RFID is however applied to many other domains and new applications appear at a very high rate, notably in health-care contexts. Actually, the pressure for a better traceability 


\section{Housseman, Absi, Feillet and Dauzère-Pérès}

in this domain (though already of obvious importance) is growing fast for several reasons: new legislations in France force a new drug pedigree using DataMatrix codes, the Activity Based Costing (ABC) principle starts being effective in French public hospitals (FHF, 2008), the USA are facing their third malpractice crisis, physicians struggling to acquire malpractice affordable insurances (Dalton, Samaropoulos, and Dalton 2008). In this context, crossing the technological pitch of using smart objects is clearly an issue. Many obvious possibilities have already been addressed like: automatic verification of the compliance to prescription, automatic checking of use-by dates, detection or confinement of devices or persons. One should also mention and insist on the role that RFID could play against drug counterfeit.

In biobanks, one can see at least two possible advantages of implementing RFID. First, automatic, acute and ubiquitous traceability of initial and finished products, devices, and mayhap persons could help reducing the number of mis-identification incidents and retrieval problems. Secondly, RFID could help to a better use the storage area. In many biobanks (at least it was true for the two biobanks involved in the project), sockets left empty because of handover requests are not reused and many boxes of cryotubes present in nitrogen tanks tend to be half-empty. It is indeed considered that re-warehousing is too risky as it might expose samples to repetitive and uncontrolled changes of temperature. RFID should permit this process. Improving these processes is all the more interesting given that the activity of biobanks seems bound to increase during the next few years: the number of cancer is growing as the population is getting older and because some recent technologies and habits may be triggering factors. Also, the number of handover requests may increase because of new techniques like toxicogenomics, the use of progenitor cells and cord-blood, or the appearance of on-line samples catalogs (e cancer.fr 2007).

As claimed previously, evaluating the impact of the integration of RFID in a logistic process is a difficult task. The literature about RFID impacts in logistic systems mainly focuses on supply chains and concerns subjects as inventory inaccuracy, bullwhip effect and replenishment policies. ROI analyses are tackled through analytical or simulation approaches (Saraç, Absi, and Dauzère-Pérès 2008b). The number of device configurations and resulting organizational scenarios is large, which is a good reason for testing some of them through simulation. The dynamic aspect of health production systems and its importance in the appearance of errors, as pictured by the "swiss cheese model" of system failures (Reason 2000), are the other main reasons that led us to using a discrete event dynamic simulation (DEDS) software. The virtual system can thus include incidents and a possibility for them to be rectified before an accident happens.

The originality of this study lies in:

- the studied production system, a complex socio-technical system where errors may occur,

- its modeling permitting the implementation of different material and organizational scenarios including smart objects,

- the study of a new activity, namely re-warehousing, allowed by the use of RFID,

- the pairing of optimization and simulation for scenarios including the re-warehousing activity in the biobanks.

Section 2 describes precisely biobank processes, insisting on the possible malfunctions and some RFID-related forecasted improvements, including the re-warehousing activity. Section 3 presents the simulation approach for this study and the new activity's optimization algorithm. Section 4 starts describing the performance indicators used, the parameters of the simulation and ends discussing the results. The last section is a conclusion leveraging future work perspectives.

\section{BIOBANK FLOW DESCRIPTION AND RFID RELATED IMPROVEMENTS}

In this section we describe the main steps undergone by a biological sample in biobanks, from sampling to shipment for final users. This description is generic and includes transformation protocols (like DNA extraction), transitory and final storages. These processes were defined together with the IPC and the CHU-N. This study allows us to precisely describe the problems that might occur during a sample processing and the potential improvements that could be achieved when introducing RFID technologies.

We will describe possible improvements when smart devices are introduced, without considering the financial impact. The main advantages of using RFID technologies instead of bar-codes are: no need of direct line of sight, a higher distance of reading, simultaneous data capture, data storage potential, and a possible resistance to harsh environments. This last point is controversial since some environments (metal, water) could prevent radio communication. For these cases, special tags and/or packaging are required.

Six main steps were identified during a sample life as depicted in Figure 1. The first one is sampling (1), including the preparation of the required tools and containers. Sampling does not necessary take place inside the biobanks but samples have to be linked to patient identity. The first process inside the biobank is the reception of the original sample (2). This sample is transformed (3), cut out or separated into multiple samples that are called aliquots. The aliquots are conserved (4) generally at a very low temperature, and sometimes inside paraffin or formalin, depending on the sample type. The next 


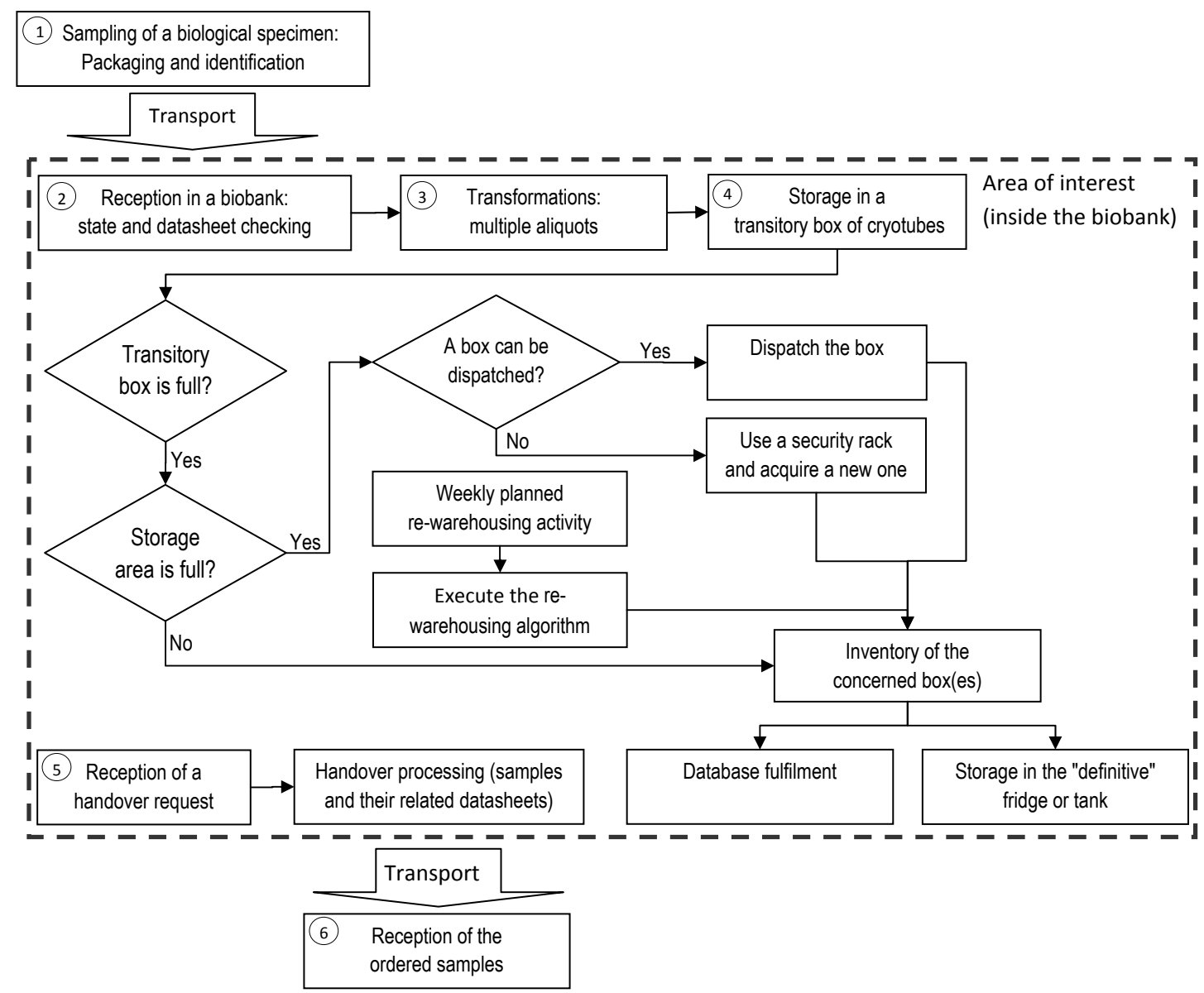

Figure 1: Biobank macro-process diagram

process is the processing of a handover request (5) that can be dedicated to a diagnosis process or a research experiment. The last process (6) is the reception of the requested aliquots by the end-user. These aliquots must be linked to the patient's information but not his identity.

In the following section, we will jointly describe in detail all the processes and the impacts that RFID could have at the operational level. The new activity of re-warehousing allowed by RFID technologies will be described in a section 2.2.

\subsection{Detailed Description of the Processes}

The transportation of a biological sample must observe specific conditions regarding transportation time, packaging and temperature. This sample can arrive through a pneumatic transportation device or be carried by a human. The current system does not allow retrieving important information, such as transportation duration, physical shocks, and cold chain failure. The data associated with the sample is also generally handwritten and is sometimes received after the biological piece, which can lead to identification errors. RFID technologies can secure this process. They allow capturing the temperature from a sensor directly linked to the sample during the transportation process. The automatic read/write function allows the traceability of the sample and then reproducing the transportation conditions (temperature, chocks, duration, etc.). A common database update allows modifying or adding information related to the sample even if it has already been sent. 


\section{Housseman, Absi, Feillet and Dauzère-Pérès}

When a sample is received in the biobank a storage ID is assigned and its state and associated data are checked. Then, the required protocols and transformations into aliquots are performed by anatomopathologist physicians and technicians. These transformations may take several days (e.g., in case of cellular culture) which may create identification errors. Generally, the differentiation between pathological and healthy aliquots is based on the color of the tube cap. We observed that tube caps could be inverted, and then create diagnosis errors that could have been avoided by saving the data directly on a transponder or in the linked database.

Once transformed and correctly packaged, the sample's aliquots are stored. In this study, we only consider the low temperature conservation case. The packages generally used are cryotubes, stocked in boxes of variable capacity. Several possibilities exist: tagging the boxes, the cryotubes, and/or the fridges.

For the time being, technicians input manually the data related to the sample and its aliquots into the Information System (IS), which is also an error prone process. Automatic information transfer using auto-ID technologies could reduce errors. At every check point, data could be sent to the IS and then provide a better traceability of the cryotubes.

With regard to step (4) of Figure 1, aliquots are first stocked in temporary boxes classed by organs and/or pathology types. Once full, these boxes are inventoried by two employees. The position of every cryotube (and of the sample it contains) is copied from the box's information sheet to the IS. This process can consume up to 40 man-minutes. With an RFID reader; the reading time can be reduced to 6 seconds, even though a basic RFID reader cannot define the position of the cryotubes in the box. Nevertheless, new prototypes (so-called X/Y antennas) allow determining the position of each cryotube and thus transferring the related information directly to the IS. Such a reader would automate the manual copy of information from the datasheet to the IS and avoid copy errors.

$\mathrm{X} / \mathrm{Y}$ antennas also gives an opportunity for a new activity within the biobank, consisting in the re-use of emptied sockets. Actually, this activity needs short and effective inventory processes. This new activity will be detailed in the next subsection.

The accuracy of the IS is especially important when a handover request is processed. If an error in a cryotube location occurs, the employee has to look for the missing cryotube at ambient temperature, which will slowly deteriorate the cryotubes. At the end-user reception point, it is important to associate every cryotube send to its information datasheet. An error during this process may distort the pharmaceutical research or make the diagnosis wrong and lead to reverse medical treatments. At the present time, the double checking principle is used at different steps to reduce all these errors. This principle reduces significantly error probabilities (Bonnabry 2007). Inserting an RFID tag to every cryotube could make the biological samples and their related information inseparable. The processes where a sample is cut-up could be done on a smart bench for the receiving cryotube's tags to be automatically updated according to the sample's one.

In the previous paragraphs, we described the main processes that a biological sample undergoes, without stretching over the different transformation protocols. RFID technologies may improve those processes at a qualitative level (error avoidance) or at a quantitative level (automated processes imply less time and resources needed). These technologies may be introduced at different levels and create new activities or change current processes, like giving up the transitory boxes or reassigning tubes between boxes.

\subsection{A New Activity: Re-warehousing}

Maintaining the stock of biological samples becomes more complex as the number of exchanges increases. In the two biobanks considered in this study, the activity of reallocating the emptied sockets was not considered since the current identification technology did not allow it and the sample exchange rate was low. The augmentation of the sample exchanges will make the reuse of emptied sockets a necessity. In this section, we describe the most realistic scenarios related to this highly constrained activity. The main constraint, stayFrozen, deals with the time limit a sample can be let out of its fridge. This constraint is the main reason why biobanks do not reallocate emptied sockets. In fact, without an appropriate technology, the inventory process is long, so the risk of deteriorating samples and also the probability of errors are high. We denote $T_{M A X}$ the maximum acceptable time of ambient temperature exposure.

Once the sample's temperature is critical, it is necessary to store it back. In our study, we do not consider the refreezing constraint. This constraint would impose that, if a box is destocked, it should not be used for a time period that must be longer than its refreezing time.

The following scenario is appropriate with the above freezing constraints. It sounds the most secure, and so the most realistic to health professionals:

- An employee takes a list of boxes out from their fridges,

- An employee moves some tubes to free a maximum number of boxes,

- An employee inventories the non-empty boxes and store them in their fridges. 


\section{Housseman, Absi, Feillet and Dauzère-Pérès}

The literature about re-warehousing activity is limited. (Garfinkel 2005) claims that (Sadiq 1993) proposes the only approach addressing the problem of optimizing the cost of picking and re-arranging warehouses in order to cluster the products. The author develops a heuristic method called "Stock Location Assignment Algorithm", this algorithm is based on the HYCLUS clustering algorithm including the possibility of moving products from a cluster to another.

The poverty of the literature about re-warehousing can be due to the fact that this process is generally done manually without any decision-support tool. Warehouse managers ask their employees to concatenate boxes and pallets when free storage areas are lacking or when human resources are available.

When using an auto-ID technology with an X/Y antenna, the inventory process is fast and accurate; so this technology should allow for efficient re-warehousing. Other scenarios could be considered for the re-warehousing activity. For example, boxes and tubes could be manipulated alternatively by more than one employee. In order to start a re-warehousing activity, we define the following possible triggers for our use case:

1. A certain amount of time has been planned for this activity (for example an hour on Fridays),

2. The overall fragmentation level of the boxes reaches a certain level,

3. A technician is unused and not supposed to be used for a certain amount of time,

4. All the tanks are full and the system needs to free some boxes (meaning rack sockets in the tanks) in order to continue working properly,

5. The system noticed that a box contains a number of tubes that is the complementary to the number of tubes in a transitory box.

Based on the preceding triggers, two main objectives could be used for re-warehousing: "Free a maximum number of boxes in a given amount of time" or "Free a given number of boxes in the minimum time length".

The general objective of this re-warehousing activity is to free as many boxes as possible. This objective can be considered in a long term or a short term strategy depending on the frequency of handover. The decisions that must be taken are: the choice of boxes to be manipulated (which boxes to be emptied and which ones to be filled), the cryotubes moves, and the order of box manipulation (this order is important since the time of exposure to ambient temperature is a strong constraint).

While introducing RFID in biobanks, many scenarios are possible since a large number of material configurations and implementation decisions are possible, plus some of them possibly allowing new activities. It is impossible to try out all these scenarios through pilot projects, since it would be expensive, potentially risk-full and hardly extensible (Royston 1999). The next section describes a decision support tool based on discrete event simulation; we also propose two re-warehousing triggers, leading to different algorithms that are implemented in the tool.

\section{SIMULATION APPROACH}

In this section we present the simulation based tool we developed. It allows decision makers to model their activities and to try out different material and organizational configurations according to the appropriate performance indicators. Our tool is based on simulation models to estimate the performance of the studied processes. (Kleijnen 2005) distinguishes four kinds of simulation that could be used for supply chain management and optimization: spreadsheet simulation, system dynamics, discrete event dynamic systems (DEDS) and business (or management) games. To implement and estimate the performance of biobank processes, we chose DEDS simulation. This choice is appropriate with the studied system since we deal with human based processes. In fact, they shall be modeled as precisely as possible to consider the Performance Shaping Factors (PSF) and errors (Park and Lee 2007). Most of healthcare processes are human or include human compounds. The use of DEDS simulation is also motivated by the demand variability and the accumulation of missed verification that can lead to errors in healthcare systems (Reason 2000). DEDS simulation can help us comparing different scenarios depending on the choice of the material and the organizational configuration. With RFID technologies we can capture realistic data that could be used to feed the simulation models (Boginski, Mun, Y. Wu, and Zhang 2007). Our work is jointly done with the IPC and CHU-N, the numerical implementations are not direct outputs from RFID technologies but are validated by health professionals.

\subsection{Model Implementation}

The developed model is designed to simply and automatically modify the needed parameters in order to try multiple scenario configurations. Every process is described by a set of three subprocesses: 


\section{Housseman, Absi, Feillet and Dauzère-Pérès}

- The most important one is the 'medical act' (sampling on patients, manipulation of samples and their cutting off, transformations, and handover preparations).

- The second subprocess is the 'Information asset'. It consists for example in writing the status (date and hour), the type and the ID of the sample, the aliquots number, etc. The use of auto-ID technologies will modify these subprocesses impacting the processing time and the associated errors probabilities.

- The last process is called 'Verification subprocess'. Errors are the most important problems in health-related production systems, so most of processes include 'Verification subprocesses'. In order to reduce these errors, a second human resource is allocated to verify the manipulator's actions. This multiple checking is supposed avoiding $85 \%$ of the incidents (Bonnabry 2007). RFID technologies can help reducing the human resource utilization and the percentage of errors.

These subprocesses are interdependent through precedence and coordination rules. For example, the 'full box storage' process contains an 'Information asset' subprocess that consists in writing the box's inventory in the IS. This subprocess needs the inventory process to be finished for the specified box. The awaited values depend on the block number and the awaiting entity's Unique IDentifier (UID).

To implement our model, we use the Arena Software described by (Law and Kelton 2000) as a general purpose simulation software, allowing the creation of customized modules, templates, and user friendly front ends. It can be easily connected with other software through ActiveX (Seppanen 2000), but also to user-coded functions through Dynamic Link Libraries (DLLs), which is useful to plug-in external algorithms that may be reused elsewhere, like in real structures for example. The model follows the macro processes described in Figure 1, where most of the links between them are a set of EntitySendToBlockLabel functions. The arriving entities are attributed a type read in the configuration file and the transformation requests sends the entity to a block named " $<$ entering entity type $>$ TO $<$ required entity type $>$ ".

A table of distances is also read in the spreadsheet of parameters; all the transfers are made through the same block, which is preceded by a VBA block configuring its parameters. In this way, an electronic information will require no human resources and a negligible delay to arrive in the computer room while a paper sheet needs a human to transport it. The time for the manipulators to reach their next task is not tallied since they do not transport any entity.

The employees are modeled using simple resources, which can be 'Available', 'Busy' or 'unreachable'. "VBA blocks" are user defined blocks used to fill the databases, assign random generated attributes like errors, the next route, process durations, or the possible number of extractable aliquots. We use these blocks to improve the way the resources are modeled and implement a basic Performance Shaping Factor (PSF) that is the visible queue length. We consider that a large amount of pending jobs increases the error rate by $5 \%$.

Process parameters can be modified between two replications through a user implemented program. These functions are implemented in VBA as recommended by (Kalsdorf 2006). Our goal is to compare material and reorganizational configurations, thus we use a spreadsheet program to define the scenarios that consists in device behaviors, process lengths and error probabilities. For example, introducing an RFID reader and appropriated software at the reception point will reduce the processing time and error probabilities. Other dysfunctions than human failures are also implemented. The inventory and information can provoke errors even if this probability is very low (device or network crashes).

The important point which is not considered in our model is the electromagnetic interferences between the RFID readers and medical machines. The results of (Togt, van Lieshout, Hensbroek, Beinat, Binnekade, and Bakker 2008) point that these incompatibility problems can make some material configurations impossible.

A virtual stock is generated for every replication, based on the transformation probabilities described in the parameter datasheet. All the scenarios start using the same initial stock files that are generated before the replications start. The triggers described in Section 2.2 could be implemented in our model. We have implemented the first and the fourth ones. The first one consists in a planned fixed process with the objective of freeing as many boxes as possible in $T_{M A X}$. The other one (emergency) aims at urgently freeing a rack socket to stock a box. The next subsection describes two algorithms allowing the execution of these activities.

\subsection{Re-warehousing Activity}

In this section we describe approaches allowing the execution of the re-warehousing activity which consists in three steps: destocking the selected boxes, moving tubes to free boxes, and then stocking the nonempty boxes in their tanks. The first decision is to select the boxes to be destocked and their ordering. The second decision is the set of tube transfers that allows boxes to be emptied. The last decision is the ordering of nonempty boxes. 


\section{Housseman, Absi, Feillet and Dauzère-Pérès}

First, we consider the simplified case where the time to destock or stock a box is not considered $\left(t_{b o x}=0\right)$. This problem can be considered as a special case of the well known knapsack problem. The objective is to select a set of boxes having the same size depending on a weight defined by the number of tubes each box contains. If $t_{b o x}=0$, we can assume that the maximal number of moves is equal to $\left\lfloor\frac{T_{M A X}}{t_{t u b}}\right\rfloor$. The maximum number of boxes that can be emptied is $\left\lfloor K-\frac{\sum_{i \in[1, K\rfloor} q_{i}}{Q}\right\rfloor$.

Property 3.1. In the particular case where $t_{b o x}=0$, the optimal algorithm to free the maximum number of boxes consists in moving the tubes from the emptiest boxes to the fullest ones.

Proof. Given $K$ boxes numbered from 1 to $K$ according to their increasing fill ratio. Suppose a solution $A$ that allows to free the $k$ first boxes and the $p^{t h}$ one. If we choose to free box $l$ instead of box $p$ such that $k<l<p$ (with $q_{k} \neq q_{l} \neq q_{p}$ ), the time needed to empty the $k$ first boxes plus box $l$ will never be larger than the time needed for solution $A$.

Property 3.2. In an optimal solution, the destocking/stocking activity must follow a FIFO rule.

Proof. Suppose that boxes are destocked in a given order $A$, and the non-empty boxes are put back in their tanks in a different order $B$, which similar to $A$, except that two boxes have been switched, namely box number $j$ will be stocked back after box number $k$. The tube manipulations start after the last box is destocked, which does not depend on the destocking and these manipulations have to stop before the time the first box is stored back. If the order $B$ is used, the box $k$ must go back in a tank at the same date as if $A$ was used. As box $j$ is re-stocked before box $k$, it advances the date the re-storing process by $t_{b o x}$ which reduces the time allowed for the tube manipulations.

Property 3.3. The best order to destock the boxes is to start by the ones that will receive tubes and to finish by the ones that will be emptied.

Proof. Considering an order $A$ where the first box that will be taken from its tank will be emptied and an order $B$ where it will receive tubes from other boxes and stocked after the manipulations. Using $A$, the content of the first box will be dispatched in one or more other boxes that will all have to be replaced before $T_{M A X}$. Using order $B$, only the first box will need to be replaced before $T_{M A X}$, so starting with the boxes that will receive tubes cannot deteriorate the solution.

The re-warehousing activity is implemented considering the two last properties. The planned process (Trigger 1) works as follows:

- The boxes are sorted in the decreasing order of their emptiness;

- The upper bound of boxes that can be emptied considering only the required number of tube manipulations is calculated;

- The maximum number of boxes that can be emptied, denoted $M$, is determined by decrementing the previous upper bound while $T_{M A X}$ is smaller than $t_{b o x}$ times $M+1$ plus the time needed to inventory the ones that will not be emptied and the time needed for the related tube manipulations.

'Trigger 4' carries out the dispatching of the box with the minimal number of tubes. If it is impossible, an emergency rack starts being used, and the time needed for the operation is set to $T_{M A X}$.

In our case study, we tried out different scenarios in biobanks, including adding RFID tags to cryotubes introducing the related improvements and the re-warehousing activity. The next section describes the studied scenarios, and shows computational results.

\section{CASE STUDY}

The model described in Section 2 is implemented. Step (1) is not precisely modeled as we consider the samples when they reach a reception area. Step (5) could be most accurately modeled, taking into account request batches similarities instead of random samples. Step (6), and the transportations taking place outside the biobank, are not implemented.

Considering technological choices, the RFID technology we want to test must ensure that cryotubes are read individually, can be put into boxes, stored in nitrogen tanks, without endanger the patient's privacy and with the possibility to read and write data. So a low-range (High Frequency, 13.56MHz) technology was chosen as a prerequisite. There are no proofs that the information in the tags will be kept if stored for years in a nitrogen tank. A study (Bettendorf, Malenfant, and Chabannon 2008) showed that it is possible to read an RFID tag that has been stored at very low temperatures $\left(-196{ }^{\circ} \mathrm{C}\right)$ for 18 months. The tested tags could not be read under the temperature of $-80^{\circ} \mathrm{C}$, but the information was intact. Accelerated physical and 


\section{Housseman, Absi, Feillet and Dauzère-Pérès}

thermal aging tests (Cauchois 2008) are currently being done at the Ecole des Mines de Saint-Etienne (some samples are stored for more than ten years...). Those first results are promising and RFID seems to be applicable at the cryotube level. The following scenarios are tested:

- "Current scenario" (Curr.Scenar), where paper sheets are associated to samples. No RFID technology is used.

- "RNA Automation" (RNA Auto.), where a RNA extraction machine is added. RNA extraction required 3 hours of manual labor, in another room at another floor than the one where the other transformations happen. The machine is as long but only requires humans when the process is started, using 20 minutes of human time for 6 to 12 sample transformations. This scenario is implemented as a part of the validation of the simulation process. Since the biobanks we work with have recently introduced these machines and are evaluating this impact, we intend to compare our results with theirs. As it is located out of the clean area of the biobank, the transportation included a time to wear the mobcap, white coat, shoe covers, and of course the time for hand wash.

- 'Smart cryotubes and pen-size antenna"(RFID \& penRder), where the data sheet is accessible from the RFID label, and the information is written in the database without the need of a manual copy of the information in the Information System. The pen-size antenna allows for the inventory of boxes by pointing all the cryotubes one after the other. We estimate that the average time needed is reduced from 24 to 8 minutes, and only uses one human resource instead of two.

- "Matrix reader's antenna" (RFID \& 2DRdr), which consists of adding the X/Y antenna, that allows for the inventory of a whole box of cryotubes recognizing their position automatically. The time needed for inventorying a box using this device is set to 2 minutes.

- "Smart cryotubes, pen-size antenna and re-warehousing"(RFID \& penRder \& RWH) is as the (RFID \& penRder) scenario where the re-warehousing activity is enabled.

- "Matrix reader's antenna and re-warehousing" (RFID \& 2DRdr \& RWH) is similar to the (RFID \& 2DRdr) scenario except that the re-warehousing activity is enabled.

The time between arrivals of new samples is exponentially distributed. The opening hours include a rush time, i.e., a time period during which the arrival rate is 1.618 times larger than during the rest of the day. These values are computed from the total number of incoming samples divided by the time the biobanks are opened yearly. The transformation protocols implemented are the DNA and RNA extractions, white-cells (that are a part of the previous ones), blood serum, plasma, and viable cells. Some of these protocols are different whether the arriving sample is blood or marrow.

The processing times of the transformations have been implemented using the data from the IPC biobank. In order to estimate the different error rates, we inventoried a few boxes randomly chosen within the stock. The results of this study are still subject to confidentiality.

The basic probability of making an error computerizing a data file by hand has been set to 1.1\%. (Marcellis-Warin 2003) indeed reports that $10 \%$ of the operations in hospitals are subject to errors and that $11 \%$ of these errors are due to retranscriptions. This error probability is set to $0.01 \%$ when tubes are tagged.

The probability that an error occurs during the inventorying of a full box of cryotubes is set to $3 \%$ when it is done by hand, which is an estimated value fixed after discussing the results of (Garnerin, Pellet-Meier, Chopard, Pergener, and Bonnabry 2007). This value has been set to $0.01 \%$ if the employees have an RFID pen size antenna to perform the inventory. These probabilities are divided by the employee "educational level". This "educational level" is the same for all the employees during a replication of the simulation; The values are either $0.5,1$, or 2 .

When re-warehousing is possible, i.e. the hardware configuration enables fast cryo-box inventories (RFID scenarios), the tactical decision of choosing triggers has been made beforehand. The first and fourth triggers have been implemented as they seem to be the most acceptable. The activity will be planned on Friday afternoons and the fourth trigger consists in emptying one box. In the latter case, the box containing the minimum number of tubes is selected for dispatching.

\subsection{Performance Indicators}

Evaluating the performance of a health service is a tall order (Minvielle, Sicotte, Champagne, Contandripoulos, Jeantet, Préaubert, Bourdil, and Richard 2007). The main points to consider are:

- the safety of patients and employees, including a hardware reliability part,

- patient satisfaction,

- financial impact, 


\section{Housseman, Absi, Feillet and Dauzère-Pérès}

- employee reluctance and/or understanding.

We consider it is inaccurate to calculate a cost related to a medical error that can possibly lead to a miscarriage of justice, or a human being's death or infection. That said, the reader can refer to (Howard, Meltzer, Kollman, Maiers, Logan, Gragert, Setterholm, and Horowitz 2005) if seeking a way to do so.

The indicators we believe are adapted to biobanks are:

Number of errors, differentiating the number of computational errors from the number of inventory errors.

Fastness of processing times, this indicator corresponds to the difference between the arriving of a new sample and the time it is computerized (IS Fulfilment delay). It could enable early fault detections. The throughput time is an interdisciplinary performance indicator which, in our case study, could make a patient obtaining his analysis results faster and its potential illness be taken care earlier.

Human utilization. We believe that the more employees use their abilities as specialists, the more they feel useful. This indicator is related to both the employee reluctance and to the financial impact. There is also a part of the customer's satisfaction in the time some health professionals like the nurses or physicians spend with the patients, even if this is not applicable within the biobanks.

Storage racks bought is the number of storage racks that had to be bought, which can be an important part of the financial indicator, depending on the storage room and tank capacity.

No formal cost calculations have been applied for many reasons: the structures may have different matters related to the increase of their storage area, some of the devices we are testing are prototypes or force the biobanks to invest in a complete proprietary solution, and the workforce costs is highly variable depending on the considered region of the world. The costs of emerging technologies decrease quickly with the increase of their use and, as the market for tagged cryotubes and readers is not very large, the prices are not stabilized yet. This indicator could be estimated by comparing the number of bought racks with the time employees spend re-warehousing the stock for this particular activity. The next section contains a larger discussion about the parameters to integer in a Return On Investment calculation.

\subsection{Results}

The scenarios described above have been tested through nine simulated months of work. Thirty-five replications of the six scenarios were ran for each of the three "educational levels" of employees. Results shown in Table 1 summarize the observed percentages of the inventory errors ("Inv. error") and computerization errors ("Comp. error"). The numbers in parenthesis corresponds to the "educational level". We did not distinguish the cases when re-warehousing is enabled for these indicators as they do not significantly differ.

Table 2 shows indicators that are not impacted by the "educational level" of the human resources in this simulation. Values given in this table indicate the improvements achieved with the different scenarios against the first one (Curr.Scenar). These indicators are IS Fulfilment delay, Human utilization and the number of storage rack bought.

The initial value, corresponding to Curr.Scenar, for the proportion of seized human resource utilization is 51.2\%, which seems right considering that the employees also spend some time working for a different service and that the amount of work to do depends on the arrival rate, which is irregular. The "worst" scenario corresponds to Curr.Scenar; the results in Table 2 are the percentage of improvement compared to this scenario.

The average number of arriving samples is about 700 per month; and the average number of information sheets computerized around 1750, including 30 new full boxes. Table 1 shows that the "educational level" has a high impact on the number of errors for the scenarios Curr.Scenar and RNA Auto.. RFID technologies remove many critical processes like handwriting and manual computerizations, so the "educational level" has less impact for the two scenarios including auto-ID.

Table 2 shows that the proportion of used resources is reduced by $10.87 \%$ when adding the RNA automaton, because RNA extraction needs less human intervention and the time needed to transport samples to the place where this transformation takes place is highly reduced as soon as the automation is in the same clean area. The gains related to this indicator when using RFID technologies are very large. The time saved there is mainly composed of computerization, handwriting and bringing information leafs. The job of the technicians would consist of tasks that are more specific to their core competences. As mentioned in the previous section, we believe this is an indicator of the employee welfare. The difference between the use of a pen reader or a matrix antenna is very low when re-warehousing is not allowed. This new activity can lead to large savings, as there is a limit for the number of nitrogen tanks that can be kept in a room. Saving a few racks may lead to saving a few tanks, which can highly impact the overall cost of maintaining the biobank. 
Table 1: Average results after 35 replications of each scenario and educational level

\begin{tabular}{|l|r|r|}
\hline $\begin{array}{l}\text { Scenario Name } \\
\text { (Ed.Level) }\end{array}$ & $\begin{array}{r}\text { Inv. } \\
\text { error }\end{array}$ & $\begin{array}{r}\text { Comp. } \\
\text { error }\end{array}$ \\
\hline Curr.Scenar(2) & $1.5 \%$ & $0.6 \%$ \\
\hline Curr.Scenar(1) & $3 \%$ & $1.2 \%$ \\
\hline Curr.Scenar(0.5) & $5.9 \%$ & $2.3 \%$ \\
\hline RNA Auto.(2) & $1.6 \%$ & $0.6 \%$ \\
\hline RNA Auto.(1) & $3 \%$ & $1.2 \%$ \\
\hline RNA Auto.(0.5) & $6 \%$ & $2.3 \%$ \\
\hline RFID \& penRder(2) & $<0.1 \%$ & $<0.01 \%$ \\
\hline RFID \& penRder(1) & $0.1 \%$ & $0.01 \%$ \\
\hline RFID \& penRder(0.5) & $0.2 \%$ & $0.02 \%$ \\
\hline RFID \& 2DRdr (2) & $0 \%$ & $<0.01 \%$ \\
\hline RFID \& 2DRdr (1) & $0 \%$ & $<0.01 \%$ \\
\hline RFID \& 2DRdr (0.5) & $<0.1 \%$ & $0.02 \%$ \\
\hline
\end{tabular}

Table 2: Proportion of human resource utilization and IS fulfilment delay compared to the current scenario, and number of storage racks used after 35 replications of each scenario

\begin{tabular}{|l|r|r|r|}
\hline Scenario Name & $\begin{array}{r}\text { Human } \\
\text { utilization }\end{array}$ & $\begin{array}{r}\text { IS fulfillment } \\
\text { delay }\end{array}$ & $\begin{array}{r}\text { Storage racks } \\
\text { bought }\end{array}$ \\
\hline Curr.Scenar & $0.00 \%$ & $0.00 \%$ & 8 \\
\hline RNA Auto. & $-10.87 \%$ & $-14.81 \%$ & 7.9 \\
\hline RFID \& penRder & $-36.74 \%$ & $-77.98 \%$ & 8 \\
\hline RFID \& 2DRdr & $-38.14 \%$ & $-78.11 \%$ & 7.9 \\
\hline RFID \& penRder\& reaff & $-36.4 \%$ & $-77.99 \%$ & 6.3 \\
\hline RFID \& 2DRdr \& reaff & $-37.9 \%$ & $-78.08 \%$ & 3.4 \\
\hline
\end{tabular}

Our results do not include a precise financial part for the reasons explained in the Section 4.2. A Return On Investment calculation must include the variation of the operating costs to the initial investments. The ratio $\frac{\text { Humanutilizationvariation }}{\text { Storageareacosts }}$, compares the time spent for re-warehousing and the savings related to a better replenishment proportion of the boxes. This investment is not necessarily linear as the limit on the number of tanks possibly kept in the storage area can be reached. The most significant parts of variable costs to consider are the variations of the cost of the (smart-)tubes (the number of tubes used does not change) and the updated maintenance costs. The main savings are the variation of the storage area and human resource costs. One would also consider the fact that insurances may reduce their fees as traceability is improved and errors reduced; so lawsuit risks concerning these biobanks are reduced. The readers, antennas, IS update, hardware, software and consulting for implementing the technology account for the fixed costs. Staff members will require an initial training concerning these technologies and associated software, as this was the case using barcodes. The use of mobile readers may notably require a time of adaptation and the processing times may evolve with their experience using it and the arrival of new hardware and software improvements (keyboards, tactile interface, graphical interface). The efficiency using new technologies evolves during time, as for example the "double labeling" (barcodes + RFID) will not be given up at first but may disappear, shall it be for being replaced by datamatrixes. These results suppose that the cryotubes are RFID-equipped, which seems quite unrealistic as it would mean replacing the tubes of existing samples or adding a smart tag on the existing ones. This last solution, if it is proved possible without deteriorating the samples, would certainly require a large investment.

\section{CONCLUSION}

In this paper, we estimated the impact of RFID technologies in a specific healthcare application with DEDS simulation used as a decision support system. Our study quantifies the benefits of integrating new auto-ID technologies and device addressing 


\section{Housseman, Absi, Feillet and Dauzère-Pérès}

one of the problematics related to this particular problem which is the possibility to enable new activities. We proposed a modeling recommendation which will be transposed in other (health) production systems.

The results show that auto-ID technologies may considerably improve the performances of biobanks when taking into account the percentages of errors as well as the reduction of resource uses and processing times as performance indicators. We still have data to analyze in order to configure the simulation and validate the relevance of some of our assumptions. This part of our work should only be possible after the pilot plants have been observed long enough so they are stabilized.

This paper addresses the re-warehousing activity, which may become a necessity in some biobanks. The proposed model should allow us to model other biobanks and/or material configurations quite quickly, including organ sample arrivals. This can be made using the same basic model file and only modifying the specific configuration file. These types of tools could be very helpful when auditing and expertizing structures that must adopt new technologies because of new legislations, or because their maximum capacity is reached for example.

Improvements could consist in taking more constraints into account for the re-warehousing processing; letting the aliquots from a sample side by side, or in the same box, could make it a much more complex problem. We are currently working on a long-term approach for re-warehousing optimization. A handover request generation based on real data would allow to improve re-warehousing, minimizing treatment times of future handovers. A graphical user interface could allow biobank managers to model their biobank. Improving the way human resources are modeled, using a multi-agent or agent-oriented systems and adding PSFs for example, would be another interesting improvement trail.

\section{ACKNOWLEDGEMENTS}

This study is partially financed by the Conseil Général 13 of the Bouches du Rhône, the MISTRALS project is funded by the MINEFE, French Ministry of Industry, Economy and Employment.

The authors gratefully thank all the collaborators of the MISTRALS project who always answered quickly and carefully to our questions, and particularly Lise-Marie Daufresne, from the IPC, and Olivier Bordonne from the CHU-N.

\section{REFERENCES}

Bettendorf, E., C. Malenfant, and C. Chabannon. 2008. RFID technologies and electronic tags to identify cryopreserved materials. In Cell preservation technology, Vol.3 (2) pp 112-114.

Boginski, V., I. Mun, K. M. Y. Wu, and C. Zhang. 2007. Simulation and analysis of hospital operations and ressource utilization using RFID data. In IEEE International Conference on RFID, Grapevine, Texas, USA, pp 199-204.

Bonnabry, P. 2007. How can the labelling and the packaging of drugs impact on the drug safety? In Swissmedic, Bern, Suisse.

Cauchois, R. 2008. Développement de tests de vieillissment accéléré de puces rfid conservées pour application en biobanques. Technical Report (undefined), Department of Packaging and Flexible Substrates., Ecole des Mines de Saint-Etienne, EMSE-CMP, 880, Route de Mimet, 13120, Gardanne (France).

Dalton, G. D., X. F. Samaropoulos, and A. C. Dalton. 2008. Improvements in the safety of patient care can help end the medical malpractice crisis in the united states. In Health Policy Vol.86, pp 153-162.

e cancer.fr 2007. <http://www.e-cancer.fr/ressources-biologiques/tumorotheque-virtuelleNationale/op_1-it_751-la_1-ve_1.html>.

Garfinkel, M. 2005. Minimizing multi-zone orders in the correlated storage assignment problem. Ph.D. thesis, Georgia Institute of Technology.

Garnerin, P., B. Pellet-Meier, P. Chopard, T. Pergener, and P. Bonnabry. 2007. Measuring human-error probabilities in drug preparation : a pilot study. In Eur J. Clin Pharmacol, Vol. 63 (8), pp 769-776.

Hedgepeth, W. O. 2007. Rfid metrics. CRC Press.

Howard, D. H., D. Meltzer, C. Kollman, M. Maiers, B. Logan, L. Gragert, M. Setterholm, and M. M. Horowitz. 2005. Optimal size of a national cord blood bank.

Kalsdorf, C. G. 2006. Clinical supply chain simulation. Ph. D. thesis, Karlsruhe University of Applied Sciences.

Kleijnen, J. 2005. Supply chain simulation tools and techniques: a survey. In International Journal of Simulation and Process Modeling, Vol.1 Nos 1/2.

Law, A., and W. Kelton. 2000. Simulation modeling and analysis. Mc Graw Series in industrial engineering and management science.

Marcellis-Warin, N. D. 2003. Les risques hospitaliers. In La lettre des cyndiniques, N40. 
Miles, S. B., S. E. Sarma, and J. R. Williams. 2008. Rfid technology and applications. The Edinburgh Building, Cambridge CB2 8RU, UK: Cambridge University Press.

Minvielle, E., C. Sicotte, F. Champagne, A.-P. Contandripoulos, M. Jeantet, N. Préaubert, A. Bourdil, and C. Richard. 2007. Hospital performance: Competing or shared values? In Health Policy, Vol. 87, Issue 1, pp 8-19.

Park, K., and J. Lee. 2007. A new method for estimating human error probabilities: Ahpslim. In Reliability Engineering \& System Safety, Vol. 93 (4), pp 578-587.

Reason, J. 2000. Human errors: Models and management. In British Medical journal, Vol.320, pp 768-770.

Royston, G. 1999. Trials versus modelling in appraising screening programmes. In British Medical journal, 318, pp 360-361.

Sadiq, M. 1993. A hybrid clustering algorithm for reconfiguration of dynamic order picking systems. Ph.D. dissertation, University of Arkansas.

Saraç, A., N. Absi, and S. Dauzère-Pérès. 2008a. A literature review on the impact of rfid techologies on supply chain management. Working Paper ENSM-SE CMP WP 2009/02.

Saraç, A., N. Absi, and S. Dauzère-Pérès. 2008b. A simulation approach to evaluate the impact of introducing rfid technologies in a three-level supply chain. In Proceedings of the 2008 Winter Simulation Conference, 2741-2749. Piscataway, New Jersey: Institute of Electrical and Electronics Engineers, Inc.

Seppanen, M. 2000. Developing industrial strength simulations models using visual basic for applications. In Proceedings of the 2000 Winter Simulation Conference, ed. S. J. Mason, R. R. Hill, L. Mnch, O. Rose, T. Jefferson, and J. W. Fowler, 77-82. Piscataway, New Jersey: Institute of Electrical and Electronics Engineers, Inc.

Togt, R. V. D., E. van Lieshout, R. Hensbroek, E. Beinat, J. Binnekade, and P. Bakker. 2008. Electromagnetic interferences from radio frequency identification including hazardous incidents in critical care medical equipment. Journal of American Medical Association 299 (24): 2884-2890.

\section{AUTHOR BIOGRAPHIES}

SYLVAIN HOUSSEMAN is Ph.D candidate in industrial engineering at the Provence Microelectronics Center of the École des Mines de Saint-Etienne, in the Manufacturing Sciences and Logistics Department. His email address for these proceedings is <houssemandemse. fr $>$.

NABIL ABSI is Associate Professor at the Provence Microelectronics Center of the École des Mines de Saint-Etienne, in the Manufacturing Sciences and Logistics Department. He received the Ph.D. degree from the Pierre and Marie Curie University (Paris 6), Paris, France, in December 2005. He was a Postdoctoral Fellow at the University of Avignon from January 2006 to August 2006. Since December 2006, he is Associate Professor at the École des Mines de Saint-Etienne. His research mostly focuses on Operations Research, optimization in production and logistics, with applications in planning, and the introduction of new communicating technologies in Supply Chains. His email address for these proceedings is $<a b s i @ e m s e$. fr $>$.

DOMINIQUE FEILLET is Professor at the Provence Microelectronics Center of the Ecole des Mines de Saint- Etienne, in the Manufacturing Sciences and Logistics Department. He received the Ph.D. degree in Industrial Engineering from the Ecole Centrale Paris, France, in 2001; and the H.D.R. in computer sciences from the University of Avignon, France, in 2007. He has been Associate Professor from 2002 to 2008 at the University of Avignon in France. Since August 2008, he is Professor at the École des Mines de Saint-Etienne. His main research interests concern the optimization of logistics processes, with a special interest on distribution and on the introduction of RFID technologies. He has published 18 papers in international journals. His email address for these proceedings is <feilleteemse.fr $>$.

STÉPHANE DAUZÈRE-PÉRÈS is Professor at the Provence Microelectronics Center of the Ecole des Mines de SaintEtienne, where he is heading the Manufacturing Sciences and Logistics Department. He received the Ph.D. degree from the Paul Sabatier University in Toulouse, France, in 1992; and the H.D.R. from the Pierre and Marie Curie University, Paris, France, in 1998. He was a Postdoctoral Fellow at the Massachusetts Institute of Technology, U.S.A., from September 1992 to December 1993, and Research Scientist at Erasmus University Rotterdam, The Netherlands, from February 1994 to July 1994. He has been Associate Professor and Professor from 1994 to 2004 at the École des Mines de Nantes in France. He was invited Professor at the Norwegian School of Economics and Business Administration, Bergen, Norway, from March 1999 to July 1999. Since March 2004, he is Professor at the École des Mines de Saint-Etienne. His research mostly focuses on optimization in production and logistics, with applications in planning, scheduling, distribution and transportation. He has published more than 20 papers in international journals and 70 communications in conferences. He is also the co-author 


\section{Housseman, Absi, Feillet and Dauzère-Pérès}

of An Integrated Approach for Production Planning and Scheduling (Springer- Verlag, 1994). His email address for these proceedings is <dauzere-pereseemse. fr $>$. 